\title{
THE UV VARIABILITY OF THE WR STAR HD 50896 - A ONE DAY RECURRENCE TIMESCALE
}

\author{
N. ST-LOUIS, A.J. WILLIS, L.J. SMITH \\ Department of Physics and Astronomy, University College London \\ Gower Street, London WC1E 6BT, U.K. \\ C.D. GARMANY, P.S. CONTI \\ Joint Institute for Laboratory Astrophysics, University of Colorado \\ Boulder CO 80309, U.S.A.
}

\section{Introduction}

The WN 5 star HD 50896 has been the subject of many variability studies in the optical. A period of 3.766 days is frequently associated with this variability although it is by no means clear whether it reflects, for example, binary motions or rotation.

In previous work ( $c f$. Willis et al. 1989) we have found substantial variations in the $\mathrm{P}$ Cygni absorption components of N V $\lambda 1240$, C IV $\lambda 1550$, He II $\lambda 1640$ and N IV $\lambda 1718$. No convincing periodicity was found and the variations were interpreted as reflecting physical changes in the velocity, density or ionization structures of the WR wind. The dataset was, however, insufficient in time resolution to accurately determine the timescale of the changes as well as any possible recurrence timescale. We have therefore obtained a further extensive time sequence of IUE spectra with the aim of gaining more insight into the nature of the changes. The preliminary results of the analysis of these new data are presented here.

\section{Observations}

In a joint NASA-Vilspa project, we have obtained a total of 130 HIRES SWP IUE spectra of HD 50896 over a period of 6 consecutive days in December 1988. The spectra were uniformly extracted from the PHOT images provided by the ground stations using the IUEDR software package available on the UK Starlink network of VAX computers. Subsequent measurements and analysis were performed using the Starlink DIPSO software package.

\section{Results}

Inspection of the spectra reveals variations in the N V $\lambda 1240, \mathrm{C}$ IV $\lambda 1550$, He II $\lambda 1640$ and N IV $\lambda 1718$ P Cygni profiles. We have quantified the changes by measuring the equivalent width of the absorption components for the last three ions (N V and C IV show a similar behaviour). The variation of the equivalent widths as a function of Julian Date is presented in Figure 1. For all three lines at least three events are clearly visible, each lasting for $\sim 1$ day, which is of the order of the flow time through the wind. The first and third events are similar for all lines while, for the second, the onset of the He II decrease seems to 
be delayed by $\sim 6$ hours. We have subjected all these measurements to a power spectrum periodicity search between $\nu_{\min }=1 /(\Delta \mathrm{t})=0.19 \mathrm{~d}^{-1}$ where $\Delta \mathrm{t}$ is the time interval over which the data were obtained and the Nyquist frequency $\nu_{\max }=\Delta \mathrm{t} / 2(\mathrm{~N}-1)=12.44 \mathrm{~d}^{-1}$ where $\mathrm{N}$ is the number of data points. The periodogram for the NIV, C IV and He II equivalent width variations show very clear peaks at $0.98,0.97$ and 1.03 days respectively.

One important characteristic of the variations is the velocity range over which they occur. This is typically between -1600 and $-2400 \mathrm{~km} \mathrm{~s}^{-1}$ for He II ; -1800 and -2800 $\mathrm{km} \mathrm{s}^{-1}$ for NIV; and -2200 and $-3150 \mathrm{~km} \mathrm{~s}^{-1}$ for CIV. This seems to reflect the line forming mechanisms of each transition and indicates that large regions of the wind are being affected at one particular time. Furthermore, most of these variations occur at velocities exceeding the bulk outflow terminal velocity of the wind $\left(\sim-1900 \mathrm{~km} \mathrm{~s}^{-1}\right)$. This is reminiscent of the radiatively driven blob model of Lucy and White (1980) or the propagating shock models of Lucy (1983) and Owocki et al. (1988).

We conclude that this extensive new dataset for HD 50896 presents the best evidence so far for intrinsic stellar wind variability, affecting large regions of the wind, and having a recurrence timescale of the order of one day.

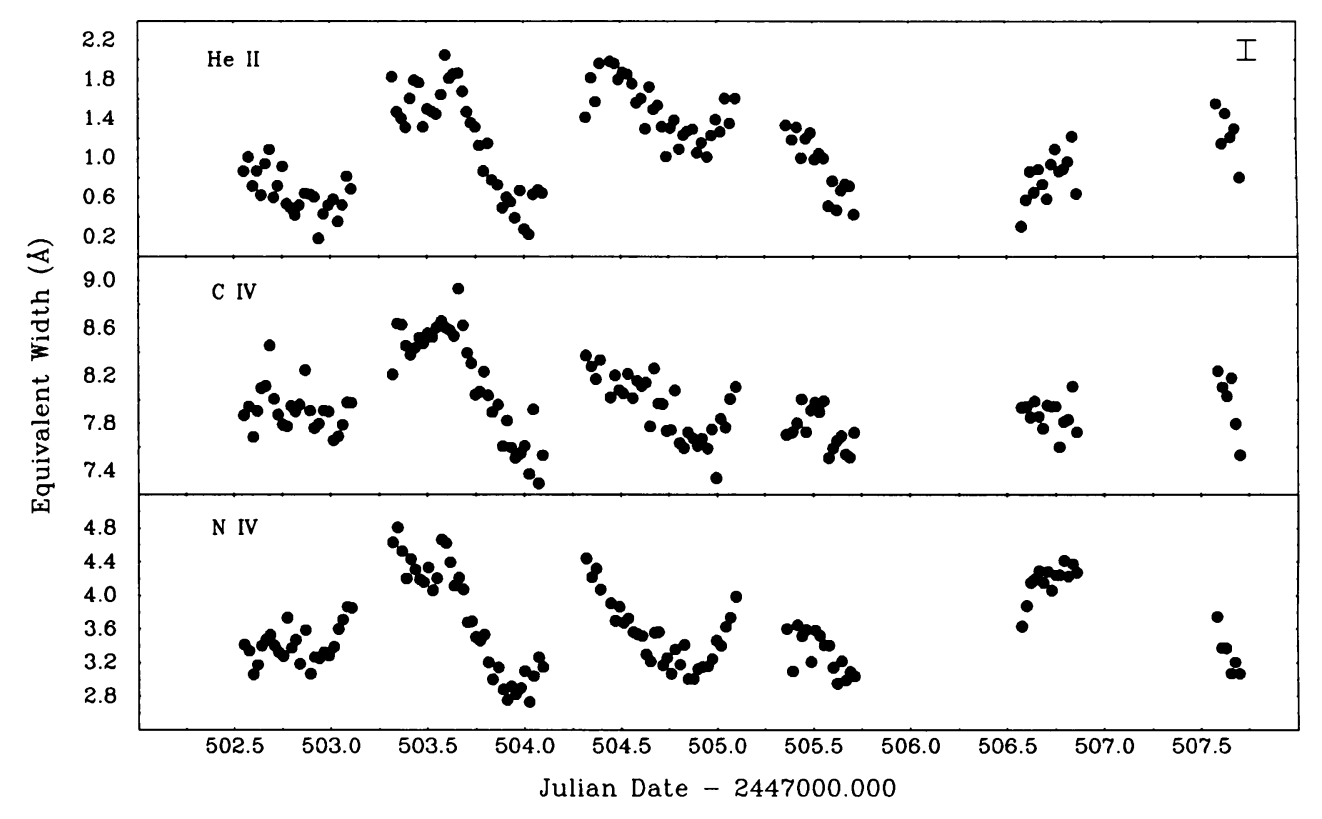

Fig. 1 : Equivalent width of absorption components as a function of Julian Date

\section{References}

Lucy, L.B. 1983, Ap. J., 274, 372.

Lucy, L.B., \& White, R.L. 1982, Ap. J., 241, 300.

Owocki, S.P., Castor, J., \& Rybicki, G.B. 1988, Ap. J., 335, 914.

Willis, A.J., Howarth, I.D., Smith, L.J., Garmany, C.D., \& Conti, P.S. 1989, Astr. Ap. Suppl., 77, 269. 